\title{
CRITICAL GLOBAL ASYMPTOTICS IN HIGHER-ORDER SEMILINEAR PARABOLIC EQUATIONS
}

\author{
VICTOR A. GALAKTIONOV
}

Received 18 October 2002

\begin{abstract}
We consider a higher-order semilinear parabolic equation $u_{t}=-(-\Delta)^{m} u-g(x, u)$ in $\mathbb{R}^{N} \times \mathbb{R}_{+}, m>1$. The nonlinear term is homogeneous: $g(x, s u) \equiv|s|^{P-1} \mathcal{s} g(x, u)$ and $g(s x, u) \equiv|s|^{Q} g(x, u)$ for any $s \in \mathbb{R}$, with exponents $P>1$, and $Q>-2 m$. We also assume that $g$ satisfies necessary coercivity and monotonicity conditions for global existence of solutions with sufficiently small initial data. The equation is invariant under a group of scaling transformations. We show that there exists a critical exponent $P=1+(2 m+Q) / N$ such that the asymptotic behavior as $t \rightarrow \infty$ of a class of global small solutions is not group-invariant and is given by a logarithmic perturbation of the fundamental solution $b(x, t)=t^{-N / 2 m} f\left(x t^{-1 / 2 m}\right)$ of the parabolic operator $\partial / \partial t+(-\Delta)^{m}$, so that for $t \gg 1, u(x, t)=C_{0}(\ln t)^{-N /(2 m+Q)}[b(x, t)$ $+o(1)]$, where $C_{0}$ is a constant depending on $m, N$, and $Q$ only.
\end{abstract}

2000 Mathematics Subject Classification: 35K55, 35K65.

1. Introduction: main results on critical global asymptotics. The main goal of the paper is to present a class of nonlinear higher-order parabolic equations with two homogeneous operators

$$
u_{t}=\mathbf{A} u+\mathbf{G}(u) \text { for } t>0, u(0)=u_{0},
$$

which are invariant relative to a group of scaling transformations, but generic global asymptotics of solutions as $t \rightarrow \infty$ are not invariants. The basic example is a $2 m$ th-order semilinear parabolic equation in the critical case, where a special nonlinear interaction between operators produces asymptotics perturbed by logarithmic factors. Such perturbed asymptotics are well known for the second-order $(m=1)$ semilinear and quasilinear heat equations and were studied in detail in the last two decades.

1.1. Statement of the asymptotic problem with critical nonlinearity. Consider the Cauchy problem for the $2 m$ th-order semilinear parabolic equation (with $m>1$ )

$$
\begin{gathered}
u_{t}=-(-\Delta)^{m} u-g(x, u) \quad \text { in } \mathbb{R}^{N} \times \mathbb{R}_{+}, \\
u(x, 0)=u_{0}(x) \text { in } \mathbb{R}^{N},
\end{gathered}
$$


where $\Delta$ denotes the Laplace operator in $\mathbb{R}^{N}$ and $u_{0} \in X=L^{1}\left(\mathbb{R}^{N}\right) \cap L^{\infty}\left(\mathbb{R}^{N}\right)$. The nonlinear term $g(x, u)$ on the right-hand side is assumed to be smooth and sufficiently small as $u \rightarrow 0$ and $g=g_{u}^{\prime}=0$ at $u=0$. Higher-order semilinear and quasilinear diffusion operators occur in applications in thin film theory, nonlinear diffusion and lubrication theory, flame and wave propagation, and phase transition at critical Lifschitz points and bistable systems (e.g., the KuramotoSivashinsky equation and the extended Fisher-Kolmogorov equation). See models and references in [30].

We are going to describe a class of (1.2) admitting a nonstandard, logarithmically perturbed asymptotic behavior as $t \rightarrow \infty$. In order to guarantee the existence of global solutions, without loss of generality of the asymptotic technique to be applied, we assume that the perturbation term $g$ satisfies a coercivity condition to ensure the existence of a local solution of the integral equation obtained by means of application of the continuous semigroup generated by $-(-\Delta)^{m}$. We thus assume that

$$
g(x, u) u \geq 0 \text { for any } u \in \mathbb{R}, x \in \mathbb{R}^{N} .
$$

Then the lower-order term is of the same sign as the diffusion operator and plays a role of an absorption-like operator ensuring the boundedness of the orbits. Multiplying equation (1.2) by $u$ and integrating by parts, this guarantees global a priori estimates on $u(\cdot, t)$ in $L^{2}\left(\mathbb{R}^{N}\right)$ and in $L^{2}\left(H^{m}\left(\mathbb{R}^{N}\right): \mathbb{R}_{+}\right)$, and semigroup techniques apply to give global solutions (see [29, 32, 33]) where detailed assumptions on nonlinearities are stated. In other applications, $g$ is a monotone operator satisfying in its domain $\langle g(u)-g(v), u-v\rangle \geq 0$, where $\langle\cdot, \cdot\rangle$ denotes the inner product in $L^{2}\left(\mathbb{R}^{N}\right)$; see examples below. We introduce the crucial assumption on the critical nonlinearity ensuring the existence of special noninvariant global asymptotics.

CRITICAL SCALING HYPOTHESIS. We assume that $g(x, u)$ is homogeneous in both variables, for all $s \in \mathbb{R}, x \in \mathbb{R}^{N}$, and $u \in \mathbb{R}$,

$$
\begin{aligned}
& g(x, s u) \equiv|s|^{P-1} s g(x, u), \\
& g(s x, u) \equiv|s|^{Q} g(x, u),
\end{aligned}
$$

with exponents $P>1$ and $Q>-2 m$. Then the critical exponent is given by

$$
P=P_{c} \equiv 1+\frac{2 m+Q}{N} .
$$

Inequality $P>1$ implies that $g_{u}^{\prime}(x, 0) \equiv 0$. In the classical case of the homogeneous algebraic nonlinearity $g(u)=|u|^{p-1} u$ with exponent $p>1$, where the semilinear equation with absorption takes the form

$$
u_{t}=-(-\Delta)^{m} u-|u|^{p-1} u \quad \text { in } \mathbb{R}^{N} \times \mathbb{R}_{+},
$$

we have $P=p, Q=0$, and the critical exponent is $P_{c}=p_{c}=1+2 m / N$. 
REMARK 1.1 (the same Fujita exponent for a blowup problem). The same exponent $p_{c}=1+2 \mathrm{~m} / \mathrm{N}$ is the critical Fujita one for the semilinear equation with source

$$
u_{t}=-(-\Delta)^{m} u+|u|^{p} \quad \text { in } \mathbb{R}^{N} \times \mathbb{R}_{+},
$$

but in the different sense, for $p \in\left(1, p_{c}\right]$, any solution $u \neq \equiv 0$ with arbitrarily small initial data satisfying $\int u_{0} \geq 0$ blows up in finite time [9, 18]. For (1.7), $p_{c}$ is exactly the critical case, where the trivial stationary solution $u \equiv 0$ loses its stability (for $p>p_{c}$ it is stable and is unstable for $p \leq p_{c}$ ). For (1.6), $u \equiv 0$ is stable for any $p>1$ and bounded solutions are always global, but their asymptotic behavior is different in the subcritical range $p<p_{c}$ and in the supercritical one $p>p_{c}$. It is worth mentioning that for any $p>1,(1.7)$ admits blowup patterns corresponding to the evolution on the centre manifold with a noninvariant logarithmically perturbed behavior [13].

For the homogeneous function $g$ containing a first-order gradient nonlinearity, we have

$$
\begin{gathered}
g(u)=|u|^{p_{0}-1} u|\nabla u|^{p_{1}}, \quad p_{0}, p_{1}>1 \Longrightarrow P=p_{0}+p_{1}, \\
Q=-p_{1} .
\end{gathered}
$$

For a more general function depending on derivatives up to $l$ th order,

$$
\begin{gathered}
g(x, u)=|x|^{\sigma}|u|^{p_{0}-1} u\left|D_{x} u\right|^{p_{1}} \ldots\left|D_{x}^{l} u\right|^{p_{l}}, \quad \sigma>-N, \\
p_{k}>1, k=0, \ldots, l<2 m,
\end{gathered}
$$

where $D_{x}^{k} u=\left\{\partial_{x}^{\beta} u:|\beta|=k\right\}, \beta=\left(\beta_{1}, \ldots, \beta_{N}\right)$ is a multi-index, $|\beta|=\beta_{1}+\cdots+$ $\beta_{N}$,

$$
P=\sum_{k=0}^{l} p_{k}, \quad Q=\sigma-\sum_{k=0}^{l} k p_{k}
$$

As a further example, we put in (1.6) an extra nonlocal multiplier via the norm in $L^{q}\left(\mathbb{R}^{N}\right)$, so that $g$ is a sufficiently smooth lower-order operator composed of linear differential or integral Hammerstein and Nemytskii operators, for example,

$$
\begin{gathered}
g(u)=|u|^{p_{0}-1} u\left(\int_{\mathbb{R}^{N}}|u|^{q} d x\right)^{v / q}, \quad v>1, q \geq 1 \Rightarrow P=p_{0}+v, \\
Q=\frac{N v}{q} .
\end{gathered}
$$

The coercivity condition (1.3) holds for (1.11). 
1.2. Main result. We will show that in the critical case $P=P_{c}$, under certain assumptions of $g$ and initial data, there exist small global solutions with the following asymptotic behavior as $t \rightarrow \infty$ :

$$
u(x, t)= \pm C_{0} t^{-N / 2 m}(\ln t)^{-N /(2 m+Q)}\left[f\left(\frac{x}{t^{1 / 2 m}}\right)+o(1)\right]
$$

where $f$ is the rescaled kernel of the fundamental solution of the linear parabolic operator (see Section 2). It is important that the constant $C_{0} \neq 0$ depends on the parameters $m, N$, and $Q$ and is independent of initial data. This critical behavior corresponds to the evolution on the local centre manifold of the nonlinear operator in the rescaled equation to be introduced in Section 4. It is a generic behavior, though there exist other types of exponentially decaying rescaled patterns on the stable manifold.

The main result for $P=P_{c}$ is proved in Section 4, where we also discuss possible generic behavior in the supercritical $P>P_{c}$ and subcritical $P \in\left(1, P_{c}\right)$ ranges.

The phenomenon of critical noninvariant asymptotics is expected to exist for a wider class of evolution equations including quasilinear higher-order parabolic equations. Nevertheless, it is not easy to specify such reasonable wellposed quasilinear models. Indeed, the invariant scaling hypothesis on the diffusion term implies that the homogeneous quasilinear operator is degenerate, for examplel, $\mathbf{A}(u)=-(-\Delta)^{m}\left(|u|^{\sigma} u\right)$ with $\sigma>0$. Uniqueness and regularity results for such degenerate equations (necessary for applying centre manifold techniques) as well as a detailed analysis of the fundamental instantaneous source-type solutions of the unperturbed equations and spectral properties of the corresponding linear non-selfadjoint operators remain open for higherorder equations with $m>1$, unlike the second-order equations, which we will discuss in a small survey below. We expect that a number of higher-order degenerate quasilinear equations with critical absorption exponents, for which proper solutions can be constructed by regularization, can admit similar noninvariant asymptotics. For instance, the phenomenon of critical noninvariant asymptotic behavior as $t \rightarrow \infty$ is expected to exist for the nonnegative solutions of the well-posed thin film equation with critical absorption in $\mathbb{R} \times \mathbb{R}_{+}$,

$$
u_{t}=-\left(u^{n} u_{x x x}\right)_{x}-u^{p} \text { with } n \in(0,3), p_{c}=n+5 .
$$

1.3. Critical behavior in nonlinear heat equations: a short survey. For the canonical semilinear heat equation with $m=1$,

$$
u_{t}=\Delta u-u^{p} \quad \text { in } \mathbb{R}^{N} \times \mathbb{R}_{+}(u \geq 0),
$$

the asymptotic behavior in the critical exponent $p_{c}=1+2 / N$ was established in $[16,15]$. For positive $L^{1}$ initial data $u_{0}(x)$ with exponential decay as $|x| \rightarrow \infty$, 
it was proved to be given by the logarithmically perturbed Gaussian kernel

$$
u(x, t)=C_{0}(t \ln t)^{-N / 2}\left[e^{-|x|^{2} / 4 t}+o(1)\right] \text { as } t \longrightarrow \infty \text { uniformly, }
$$

where $C_{0}$ is calculated explicitly, $C_{0}=(N / 2)^{N / 2}(1+2 / N)^{N^{2} / 2}$. Compactness of the rescaled orbits was proved by constructing suitable super- and subsolutions having a structure similar to (1.15) (an estimate from below was earlier obtained in [23]), and the uniqueness of the rescaled limit (uniqueness of the constant $C_{0}$ ) was proved by a special energy analysis of the rescaled equation.

A principle generalization was obtained in [4, 5], where such logarithmically perturbed asymptotics were justified by a perturbation analysis of linearized second-order selfadjoint operator and, hence, were shown to exist for a wide class of second-order semilinear evolution equations.

Logarithmic factors in the asymptotics can occur for (1.14) due to a different mechanism; for instance, for $p>p_{c}$, assuming that the initial function has a critical asymptotics $\sim|x|^{-N}$ as $|x| \rightarrow \infty$ [25]. It is a phenomenon of interaction of the Laplacian with initial data (having a critical behavior at infinity), which follows from the convolution $b(t) * u_{0}$.

The critical asymptotic behavior of nonnegative solutions exists for secondorder quasilinear heat equations like the porous medium equation (PME) with absorption

$$
u_{t}=\Delta|u|^{\sigma} u-|u|^{p-1} u \text { in } \mathbb{R}^{N} \times \mathbb{R}_{+}, \sigma>0, p>1 .
$$

The critical exponent is $p_{c}=\sigma+1+2 / N$, and for compactly supported initial data $u_{0}$, the critical behavior as $t \rightarrow \infty$ takes the form

$$
u(x, t)=(t \ln t)^{-\gamma}\left[f_{*}\left(x t^{-\gamma / N}(\ln t)^{\gamma \sigma / 2}\right)+o(1)\right], \quad \gamma=\frac{N}{N \sigma+2},
$$

where $f_{*} \geq 0$ is a uniquely chosen rescaled profile of the famous Zel'dovichKompaneetz-Barenblatt similarity solution of the PME $u_{t}=\Delta|u|^{\sigma} u$. It has the form

$$
f_{*}(y)=\left[\frac{\gamma \sigma}{2 N(\sigma+1)}\left(a_{*}^{2}-|y|^{2}\right)_{+}\right]^{1 / \sigma},
$$

where $(\cdot)_{+}$denotes the positive part. The constant $a_{*}$, playing a role of $C_{0}$ in the semilinear case (1.15), is uniquely determined as follows:

$$
a_{*}=\left[\frac{2 N(\sigma+1)}{\gamma \sigma}\right]^{1 / 2}\left[\frac{N B(N / 2,1+1 / \sigma)}{2 B\left(N / 2,1+p_{c} / \sigma\right)}\right]^{\gamma \sigma / 2},
$$

with $B(\cdot, \cdot)$ being Euler's Beta function. Unlike the semilinear case $\sigma=0$, in (1.17) an unbounded $\ln t$-factor scales also the space variable. Proof of convergence (1.17) was done in [19] for the one-dimensional case $N=1$, where 
the rescaled equation was shown to admit an approximate Lyapunov function being "almost" monotone on evolution orbits (compactness of the rescaled orbits via super- and sub-solutions as for $\sigma=0$ and the uniqueness of the asymptotic limit were established for any $N \geq 1$ ). The proof of (1.17) for any $N>1$ was done in [21] by a general stability approach for perturbed dynamical systems with uniformly stable $\omega$-limit sets. Similar $\ln t$-perturbed asymptotics are available for the $p$-Laplacian equation with the critical absorption

$$
\begin{gathered}
u_{t}=\nabla \cdot\left(|\nabla u|^{\sigma} \nabla u\right)-|u|^{p-1} u, \quad \sigma>0, \\
p_{c}=\sigma+1+\frac{\sigma+2}{N} .
\end{gathered}
$$

Compactness of the rescaled orbits and the uniqueness of the limit profile were established in [19], and passage to the limit was performed in [21] via a dynamical systems approach. We note that linearization techniques similar to those in $[4,5]$ are not straightforward for quasilinear equations like (1.16) and (1.20). A linearization procedure about compactly supported profiles like (1.18) even for $N=1$ leads to a singular second-order symmetric ordinary differential operators on bounded intervals (unlike the semilinear case $\sigma=0$ ) having singularities at finite endpoints. Spectral analysis and completeness of eigenfunctions for suitable selfadjoint extensions of such operators are not straightforward. For $N>1$, one obtains complicated problems on selfadjoint extensions of singular elliptic operators. Further results on asymptotics of quasilinear heat equations with absorption can be found in ([31, Chapters 2 and 4$]$ and the references therein).

Logarithmically perturbed "dipole” Barenblatt-Zel'dovich similarity solutions for the PME with absorption $(u \geq 0)$

$$
u_{t}=\left(u^{m}\right)_{x x}-u^{p} \quad \text { in } \mathbb{R}_{+} \times \mathbb{R}_{+},\left.u\right|_{x=0} \equiv 0,
$$

$m>0, p_{c}=m+1$, were studied in [20]. Critical absorption exponents cannot be calculated explicitly if the corresponding unperturbed equation admits the generic behavior described by self-similarity of the second kind, which cannot be found via a dimensional analysis. For example, this is true for the $1 \mathrm{D}$ dual PME $u_{t}=\left|u_{x x}\right|^{m-1} u_{x x}$, where $m>1$ (see [1]). The critical absorption exponent for the dual PME with absorption

$$
u_{t}=|\Delta u|^{m-1} \Delta u-|u|^{p-1} u \quad \text { in } \mathbb{R}^{N} \times \mathbb{R}_{+}, m>1, p>1
$$

is calculated but cannot be explicitly expressed via the diffusion exponent $m$ and dimension $N$ [14]. The critical Fujita exponent for the one-dimensional dual PME with the source

$$
u_{t}=\left|u_{x x}\right|^{m-1} u_{x x}+u^{p}, \quad m>1, p>1
$$

was calculated in [17]. 
2. Preliminaries: fundamental solution and semigroup. We consider classical solutions of the Cauchy problem satisfying the integral equation

$$
u(t)=e^{-(-\Delta)^{m} t} u_{0}-\int_{0}^{t} e^{-(-\Delta)^{m}(t-s)} g(u(s)) d s, \quad t>0 .
$$

Let $p(\omega)=-|\omega|^{m}$ be the characteristic polynomial of $-(-\Delta)^{m}$. Then $e^{-(-\Delta)^{m} t} u_{0}$ $=b(t) * u_{0}$, where the kernel $b(x, t)$ of the integral operator $e^{-(-\Delta)^{m} t}$ is the fundamental solution of the parabolic operator $\partial / \partial t+(-\Delta)^{m}$,

$$
b(x, t)=\mathscr{F}^{-1}\left(e^{p(\omega) t}\right) \equiv(2 \pi)^{-N} \int_{\mathbb{R}^{N}} e^{-|\omega|^{m} t-i(\omega \cdot x)} d \omega \quad(b(x, 0)=\delta(x)) .
$$

It follows that it takes the standard self-similar form

$$
b(x, t)=t^{-N / 2 m} f(y), \quad y=\frac{x}{t^{1 / 2 m}} .
$$

Substituting $b(x, t)$ into the linear equation

$$
u_{t}=-(-\Delta)^{m} u,
$$

by the uniqueness of the fundamental solution of linear differential operators, the radially symmetric profile $f(y)$ is a unique solution of a linear ordinary differential equation (ODE), which is the radial restriction of the elliptic equation

$$
\mathbf{B} f \equiv-\left(-\Delta_{y}\right)^{m} f+\frac{1}{2 m} \nabla_{y} f \cdot y+\frac{N}{2 m} f=0 \quad \text { in } \mathbb{R}^{N}, \int_{\mathbb{R}^{N}} f=1 .
$$

The operator $\mathbf{B}$ has the divergent representation

$$
\mathbf{B} f \equiv-(-\Delta)^{m} f+\frac{1}{2 m} \nabla \cdot(y f) .
$$

For $m>1$, the rescaled kernel changes sign and $f=f(\xi)$, where $\xi=|y|$, is oscillating as $\xi \rightarrow \infty$. Estimates of fundamental solutions, their derivatives, and other properties are available in [11]. In particular, it is convenient to present an upper estimate of $f$ in the following form: there exist constants $D>1$ and $d>0$ depending on $m$ and $N$ such that

$$
|f(y)|<D F(y) \equiv D \omega_{1} e^{-d|y|^{\alpha}} \quad \text { in } \mathbb{R}^{N}, \alpha=\frac{2 m}{2 m-1} \in(1,2),
$$

where $\omega_{1}>0$ is a normalization constant such that $\int F=1$. The positive kernel

$$
\bar{b}(x, t)=t^{-N / 2 m} F(y), \quad \int \bar{b}(x, t) d x \equiv 1,
$$

is then the majorizing one for $b$ in the sense that $|b(x, t)| \leq D \bar{b}(x, t)$ in $\mathbb{R}^{N} \times \mathbb{R}_{+}$. Therefore, solutions of order-preserving integral equation with the 
majorizing kernel $\bar{b}$ can be compared with solutions of the original PDEs like (2.1) and this gives global existence of small solutions of (1.7) for $p>1+2 \mathrm{~m} / \mathrm{N}$ and estimates on blowup rates of general solutions (see [6, 18]). Local and global solvability and regularity properties of classical solutions of

$$
u(t)=b(t) * u_{0}-\int_{0}^{t} b(t-s) * g(u(s)) d s
$$

are well known (see, e.g., [33, Chapter 15] and recent results in [7, 10, 18]).

3. Spectral properties of B and of the adjoint operator B*. We begin with the spectral properties of $\mathbf{B}$ and the corresponding adjoint operator $\mathbf{B}^{*}$ which will play a key role in further asymptotic analysis of the nonlinear problem.

3.1. Point spectrum of non-selfadjoint operator B. For $m>1$, B is not symmetric and does not admit a selfadjoint extension. We consider $\mathbf{B}$ in the weighted space $L_{\rho}^{2}\left(\mathbb{R}^{N}\right)$ with the exponentially growing weight function

$$
\rho(y)=e^{a|y|^{\alpha}}>0 \quad \text { in } \mathbb{R}^{N},
$$

where $a \in(0,2 d)$ is a constant. We ascribe to $\mathbf{B}$ the domain $H_{\rho}^{2 m}\left(\mathbb{R}^{N}\right)$. The following result is valid [10,13].

LEMMA 3.1. (i) The operator $\mathbf{B}: H_{\rho}^{2 m}\left(\mathbb{R}^{N}\right) \rightarrow L_{\rho}^{2}\left(\mathbb{R}^{N}\right)$ is a bounded linear operator with only the real point spectrum

$$
\sigma(\mathbf{B})=\left\{\lambda_{\beta}=-\frac{|\beta|}{2 m},|\beta|=0,1,2, \ldots\right\} .
$$

Eigenvalues $\lambda_{\beta}$ have finite multiplicity with eigenfunctions

$$
\psi_{\beta}(y)=\frac{(-1)^{|\beta|}}{\sqrt{\beta !}} D^{\beta} f(y)
$$

(ii) The set of eigenfunctions $\Phi=\left\{\psi_{\beta},|\beta|=0,1,2, \ldots\right\}$ is complete in $L_{\rho}^{2}\left(\mathbb{R}^{N}\right)$.

(iii) The operator $\mathbf{B}$ is sectorial in $L_{\rho}^{2}$ and in $l_{\rho}^{2}$.

The "little" $L^{2}$-space $l_{\rho}^{2} \subset L_{\rho}^{2}\left(\mathbb{R}^{N}\right)$ consists of functions $v=\sum a_{\beta} \psi_{\beta}$ with coefficients $\left\{a_{\beta}\right\} \in l^{2}$, that is, $\sum a_{\beta}^{2}<\infty$ with the same inner product [10]. In the classical second-order case $m=1, f(y)=(4 \pi)^{-N / 2} e^{-|y|^{2} / 4}$ is the rescaled positive Gaussian kernel and the eigenfunctions are

$$
\psi_{\beta}(y)=e^{-|y|^{2} / 4} H_{\beta}(y), \quad H_{\beta}(y) \equiv H_{\beta_{1}}\left(y_{1}\right) \cdots H_{\beta_{N}}\left(y_{N}\right),
$$

where $H_{\beta}$ are Hermite polynomials in $\mathbb{R}^{N}$ [2]. Operator $\mathbf{B}$ with the domain $H_{\rho}^{2}\left(\mathbb{R}^{N}\right)$ with the weight $\rho=e^{|y|^{2} / 4}$ is selfadjoint and the eigenfunctions form an orthogonal basis in $L_{\rho}^{2}\left(\mathbb{R}^{N}\right)$. 
The point spectrum of $\mathbf{B}$ is calculated by differentiating the elliptic equation (2.5),

$$
D^{\beta} \mathbf{B} f=\mathbf{B} D^{\beta} f+\frac{|\beta|}{2 m} D^{\beta} f=0 .
$$

Performing the rescaling $u(x, t)=t^{-N / 2 m} w(y, \tau), y=x / t^{1 / 2 m}$ and $\tau=\ln t \in$ $\mathbb{R}$ of a solution $u(x, t)$ of (2.4) with initial data $u_{0} \in H_{\rho}^{2 m}\left(\mathbb{R}^{N}\right)$, yields the parabolic equation

$$
w_{\tau}=\mathbf{B} w \quad \text { for } \tau=\ln t \in \mathbb{R} \text {. }
$$

Rescaling the convolution $u(t)=b(t) * u_{0}$ leads to the following explicit representation of the semigroup $e^{\mathbf{B} \tau}$ :

$$
w(y, \tau)=\int_{\mathbb{R}^{N}} f\left(y-z e^{-\tau / 2 m}\right) u_{0}(z) d z,
$$

and further Taylor expansion in the kernel shows that (3.2) is the point spectrum. Completeness of $\Phi$ is proved in [10] by the Riesz-Fischer theorem which is similar to the completeness of Hermite's or Laguerre's orthogonal polynomials (see [27, page 431]). Completeness is also associated with exact semigroup representation (3.7) (no other eigenfunctions from $L_{\rho}^{2}\left(\mathbb{R}^{N}\right)$ can occur in the expansion).

Operator $\tilde{\mathbf{B}}=\mathbf{B}-I$ has the strictly negative point spectrum $\sigma_{p}(\tilde{\mathbf{B}})=\left\{\tilde{\lambda}_{\beta}=\right.$ $-1-|\beta| / 2 m\}$. By the explicit convolution representation (3.7), the descent method of construction of fundamental solutions implies that

$$
\tilde{\mathbf{B}}^{-1} \boldsymbol{g} \equiv K * g, \quad g \in L_{\rho}^{2}\left(\mathbb{R}^{N}\right),
$$

with the kernel

$$
K(y, \zeta)=-\int_{0}^{1}(1-z)^{-N / 2 m} f\left[\left(y-\zeta z^{1 / 2 m}\right)(1-z)^{-1 / 2 m}\right] d z .
$$

In view of known oscillatory properties of the exponentially decaying rescaled kernel $f$, see (2.7), using a transformation $\mathbb{R}^{N} \rightarrow B_{1}$ in both independent variables in (3.9) $\left(B_{1}\right.$ is the unit ball), we have that $K$ is an $L^{p}$-kernel, $p \in(1,2]$, and (3.8) is a compact operator with a discrete spectrum accumulating at 0 . Thus, B has only a point spectrum, the resolvent $(\mathbf{B}-\lambda I)^{-1}$ in $l_{\rho}^{2}$ has a pole $\sim 1 / \lambda$ as $\lambda \rightarrow 0$ ( $\lambda_{0}=0$ has a multiplicity one) [24], and $\mathbf{B}$ is sectorial [12].

Lemma 3.1 gives the centre and stable subspaces of $\mathbf{B}, E^{c}=\operatorname{Span}\left\{\psi_{0}=f\right\}$ and $E^{\mathcal{S}}=\operatorname{Span}\left\{\psi_{\beta},|\beta|>0\right\}$.

3.2. Spectrum and polynomial eigenfunctions of the adjoint operator $B^{*}$. We now describe eigenfunctions of the adjoint operator

$$
\mathbf{B}^{*}=-(-\Delta)^{m}-\frac{1}{2 m} y \cdot \nabla .
$$


In the second-order case $m=1$, it admits a symmetric representation

$$
\mathbf{B}^{*}=\frac{1}{\rho^{*}} \nabla \cdot\left(\rho^{*} \nabla\right), \quad \rho^{*}(y)=e^{-|y|^{2} / 4} .
$$

Then $-\mathbf{B}^{*} \geq 0$ is semibounded and there exists its unique Friedrichs extension, which is a selfadjoint operator in the weighted Hilbert space $L_{\rho^{*}}^{2}\left(\mathbb{R}^{N}\right)$ with the domain $\mathscr{D}\left(\mathbf{B}^{*}\right)=H_{\rho^{*}}^{2}\left(\mathbb{R}^{N}\right)$ and a discrete spectrum. The eigenfunctions form an orthonormal basis in $L_{\rho^{*}}^{2}\left(\mathbb{R}^{N}\right)$ and the classical Hilbert-Schmidt theory applies (see [2]).

Let $m>1$ and consider $\mathbf{B}^{*}$ in $L_{\rho^{*}}^{2}\left(\mathbb{R}^{N}\right)$ with the exponentially decaying weight function

$$
\rho^{*}(y)=\frac{1}{\rho(y)} \equiv e^{-a|y|^{\alpha}}>0 .
$$

The following results are valid [10].

LEMmA 3.2. (i) The operator $\mathbf{B}^{*}: H_{\rho^{*}}^{2 m}\left(\mathbb{R}^{N}\right) \rightarrow L_{\rho^{*}}^{2}\left(\mathbb{R}^{N}\right)$ is a bounded linear operator with spectrum $\sigma\left(\mathbf{B}^{*}\right)$ given by (3.2). Eigenfunctions $\psi_{\beta}^{*}(y)$ are $|\beta|$ thorder polynomials

$$
\psi_{\beta}^{*}(y)=\frac{1}{\sqrt{\beta !}}\left[y^{\beta}+\sum_{j=1}^{[|\beta| / 2 m]} \frac{1}{j !}(-\Delta)^{m j} y^{\beta}\right] .
$$

(ii) The subset $\left\{\psi_{\beta}^{*}\right\}$ is complete in $L_{\rho^{*}}^{2}\left(\mathbb{R}^{N}\right)$.

(iii) The operator $\mathbf{B}^{*}$ is sectorial in $L_{\rho^{*}}^{2}$ and $l_{\rho^{*}}^{2}$.

With this definition of the adjoint eigenfunctions, the orthonormality condition holds:

$$
\left\langle\psi_{\beta}, \psi_{\gamma}^{*}\right\rangle=\delta_{\beta, \gamma} .
$$

We use the expansion analysis of the explicit convolution representation. In order to get the adjoint operator $\mathbf{B}^{*}$, we introduce different rescaled variables corresponding to blowup as $t \rightarrow 1^{-}, u(x, t)=w(y, \tau), y=x /(1-t)^{1 / 2 m}$, and $\tau=-\ln (1-t) \quad(0<t<1)$, and then $w$ solves the problem

$$
w_{\tau}=\mathbf{B}^{*} w \text { for } \tau>0, \quad w(0)=u_{0} .
$$

Rescaling the convolution $u(t)=b(t) * u_{0}$ yields the explicit representation of the semigroup with the infinitesimal generator $\mathbf{B}^{*}$

$$
w(y, \tau)=\int_{\mathbb{R}^{N}} f(\zeta-v) u_{0}\left(\zeta t^{1 / 2 m}\right) d \zeta, \quad v=y[(1-t) / t]^{1 / 2 m}
$$

The asymptotic expansions in (3.16) as $\tau \rightarrow \infty\left(t \rightarrow 1^{-}\right)$gives a complete point spectrum in $L_{\rho^{*}}^{2}\left(\mathbb{R}^{N}\right)$, (see [13]). Completeness follows from (3.13) and the wellknown fact that polynomials $\left\{y^{\beta}\right\}$ are complete in $L^{p}$-spaces with any suitable 
positive weights. Regardless of the pure polynomial structure of eigenfunctions, completeness properties in weighted spaces can be seen from the continuity of the corresponding uniformly parabolic flow (3.15). For $m=1$, both (3.2) and (3.13) are well-known properties of the separable Hermite polynomials generated by a selfadjoint Sturm-Liouville problem [2].

Taking the operator $\mathbf{B}^{*}-I$ with uniformly negative point spectrum and using the fact that operations $(\cdot)^{*}$ and $(\cdot)^{-1}$ commute for operators in Banach spaces, and that adjoint operator of a compact operator is compact, we have that $\left(\mathbf{B}^{*}-I\right)^{-1}$ is compact with only the point spectrum.

4. Centre manifold behavior: the main result. It is convenient to state the main result in terms of rescaled variables generated by the similarity structure of the fundamental solution. We perform the change of the dependent and independent variables $(u, x, t) \mapsto(v, y, \tau)$, where

$$
\begin{gathered}
u(x, t)=(1+t)^{-N / 2 m} v(y, \tau), \\
y=\frac{x}{(1+t)^{1 / 2 m}}, \tau=\ln (1+t): \mathbb{R}_{+} \longrightarrow \mathbb{R}_{+} .
\end{gathered}
$$

The critical exponent $P_{c}$ has been chosen in such a way that, under the scaling invariance condition (1.4) with $P=P_{c}$, the scaling group admitted by the full equation (1.2) is the same as the group of the linear equation (2.4). Therefore, in terms of the new rescaled variables (4.1) we obtain an autonomous (timeindependent) parabolic equation

$$
v_{\tau}=\mathbf{A}(v) \equiv \mathbf{B} v-g(y, v) \quad \text { for } \tau>0, v(0)=u_{0}
$$

We consider sufficiently small initial data $u_{0} \in H_{\rho}^{2 m}\left(\mathbb{R}^{N}\right)$ satisfying $\left|u_{0}(y)\right| \leq$ $c e^{-b|y|^{\alpha}}$ in $\mathbb{R}^{N}$, where $c>0$ is small and $b \geq d$ is large enough.

Sectorial operator $\mathbf{B}$ generates a strong continuous analytic semigroup $\left\{e^{\mathbf{B} \tau}\right.$, $\tau \geq 0\}$ (see [12]). The asymptotic behavior with a finite-dimensional local centre manifold is covered by the invariant manifold theory (see [28, Chapter 6]) using interpolation spaces $E_{i}=D_{\mathbf{B}}(\theta+i, \infty)$ for $i=0,1, \theta \in(0,1)$. The main assumption on the spectral set $\sigma_{+}(\mathbf{B})=\{\lambda \in \sigma(\mathbf{B}): \operatorname{Re} \lambda \geq 0\}$ is valid, and moreover, $\sigma_{+}$(B) consists of a unique zero simple eigenvalue $\lambda_{0}=0$ with the eigenfunction $\psi_{0}=f$ (no unstable subspace is available). Setting $\sigma_{-}(\mathbf{B})=\{\lambda \in$ $\sigma(\mathbf{B}): \operatorname{Re} \lambda<0\}$, we observe a positive gap

$$
\omega_{-}=-\sup \left\{\operatorname{Re} \lambda: \lambda \in \sigma_{-}(\mathbf{B})\right\}=\frac{1}{2 m}>0 .
$$

Using projection $P$ associated with the spectral set $\sigma_{+}(\mathbf{B}), P\left(E_{0}\right) \subset E_{1}$, leads to a one-dimensional equation for $X(\tau)=P v(\tau)$,

$$
X^{\prime}=\mathbf{B}_{+} X-P g(X+Y), \quad \tau \geq 0,
$$


where $\mathbf{B}_{+}=\left.\mathbf{B}\right|_{P\left(E_{0}\right)}$ is the null operator (since $\lambda_{0}=0$ ) and $Y(\boldsymbol{\tau})=(I-P) v(\boldsymbol{\tau})$. Necessary assumptions on the nonlinear term $g$ are valid for several kinds of such lower-order operators, see the conditions in [28, Section 9.2]. Various projectivity methods for non-selfadjoint cases can be found in [32]. It then follows from [28, Theorem 9.2.2] that there exists a one-dimensional invariant local centre manifold $W^{c}(0)$ of the origin, which is the graph of a Lipschitz continuous function $\gamma: P\left(E_{0}\right) \rightarrow(I-P)\left(E_{1}\right)$. Moreover, it follows from (4.3) that it is exponentially attractive provided that $g$ is twice continuously differentiable, see [28, Proposition 9.2.3]. Thus, we state the following condition on $\mathfrak{g}$ :

$$
\text { there exists a one-dimensional } W_{\text {loc }}^{c}(0) \text {. }
$$

Under the above hypotheses, we have the following result.

THEOREM 4.1. Let (1.4) be valid with the critical exponent $P=P_{c} \geq 2$ given in (1.5). Let twice continuously differentiable function $g(\cdot, v)$ be such that (4.5) holds and

$$
R_{*} \equiv\left\langle g(\cdot, f), \psi_{0}^{*}\right\rangle>0,
$$

where $\psi_{0}^{*} \equiv c_{0}^{*}>0$ is the first eigenfunction of the adjoint operator $\mathbf{B}^{*}$. Then any small solution $v(\cdot, \tau)$, which does not decay exponentially fast, has the following asymptotic behavior as $t \rightarrow \infty$ :

$$
v(y, \tau)= \pm C_{0} \tau^{-N /(2 m+Q)}[f(y)+o(1)], \quad \text { where } C_{0}=\left[\frac{R_{*}(2 m+Q)}{N}\right]^{-N /(2 m+Q)}
$$

Hence, (4.7) implies that the null solution is asymptotically stable in $E_{1}$ (see [28, page 371]).

Proof. The projection is $P v=\left\langle v, \psi_{0}^{*}\right\rangle \psi_{0}$ with $\psi_{0}=f$ and $\psi_{0}^{*} \equiv 1$. The behavior of the local centre manifold is given by the one-dimensional equation (see [28, pages 365-371]) $z^{\prime}(\tau)=P g(z(\tau)+\gamma(z(\tau)))$ for $\tau \geq 0$, where by the regularity assumptions on the nonlinearity, $\gamma^{\prime}(0)=0$. Setting $z(\tau)=a_{0}(\tau) \psi_{0}$, we have

$$
a_{0}^{\prime}=-\left\langle g\left(a_{0} \psi_{0}+o\left(a_{0}\right)\right), \psi_{0}^{*}\right\rangle
$$

Using the second homogenuity hypothesis in (1.4), we finally derive the evolution equation of the local centre manifold

$$
a_{0}^{\prime}=-\left|a_{0}\right|^{P-1} a_{0}\left\langle g\left(\psi_{0}\right), \psi_{0}^{*}\right\rangle+o\left(\left|a_{0}\right|^{P}\right) \equiv-R_{*}\left|a_{0}\right|^{P-1} a_{0}+o\left(\left|a_{0}\right|^{P}\right) .
$$

In the derivation, we have used that $\psi_{0}(y)$ is exponentially decaying as $|y| \rightarrow$ $\infty$ and $g(\cdot, v)=O\left(|v|^{P}\right)$ as $v \rightarrow 0$. Equation (4.9) can be integrated asymptotically as a standard ODE and admits only globally decaying orbits (4.7). 
REMARK 4.2 (unstable centre manifold behavior). The sign restriction (4.6) is essential. If $R_{*}<0$, then the asymptotic ODE (4.9) implies unstability of the origin via centre manifold evolution. In the case of (1.7) with nonnegative nonmonotone perturbation, this is exactly the case: any solution with initially positive first Fourier coefficient, $\int u_{0}>0$, blows up in finite time (see different proofs in [9] (by a test-function method) and in [18] (by a modification of Kaplan's eigenfunction method)).

REMARK 4.3 (exponentially decaying patterns on the stable manifold). Concerning another assumption of the theorem, we note that general equation (4.2) admits orbits on the infinite-dimensional stable manifold of the origin, which follows from the eigenfunctions expansion of solutions. Under natural hypotheses on nonlinear term $g, v(y, \tau)$ for $\tau \geq 0$ is sufficiently smooth by the parabolic regularity theory (see [11, 12]). In view of completeness and orthonormality of eigenfunctions of $\mathbf{B}$, for smooth small initial data $v_{0} \in H_{\rho}^{2 m}\left(\mathbb{R}^{N}\right)$, we use the eigenfunctions expansion of the solution

$$
v(\tau)=\sum_{\beta} a_{\beta}(\tau) \psi_{\beta}=a_{0}(\tau) \psi_{0}+\sum_{|\beta| \geq 1} a_{\beta}(\tau) \psi_{\beta} \equiv X(\tau)+Y(\tau),
$$

where $X(\tau) \equiv P v(\tau) \in E^{c}$, and $Y(\tau) \in E^{s}$ for all $\tau>0$ are the corresponding projections. The expansion coefficients satisfy the dynamical system

$$
a_{\beta}^{\prime}=\lambda_{\beta} a_{\beta}-\left\langle g(\cdot, v), \psi_{\beta}^{*}\right\rangle \text { for any } \beta,
$$

where the first equation with $|\beta|=0$ gives the evolution equation on the onedimensional local centre manifold. The diagonal structure of the system (4.11) shows that if the nonlinear term $g$ forms an exponentially decaying perturbation as $\tau \rightarrow \infty$, then there exist patterns with exponential decay as $\tau \rightarrow \infty$

$$
v(y, \tau)=C e^{\lambda_{\beta} \tau}\left(\psi_{\beta}(y)+o(1)\right), \quad C=C\left(u_{0}\right) \neq 0,
$$

where $\psi_{\beta}$ is a suitable eigenfunction with $\lambda_{\beta}<0$ for $|\beta|>0$. Indeed, asymptotically, these are exponentially decaying solutions of the linear equation (3.6). Such results are well known in the linear perturbation theory, (see [8, 12]).

4.1. Asymptotic behavior in the supercritical range. Assuming that $P>P_{c}$ and performing rescaling (4.1), we obtain a perturbed equation

$$
v_{\tau}=\mathbf{B} v-e^{\gamma \tau} g(y, v), \quad \text { where } \gamma=\frac{N\left(P_{\mathcal{C}}-P\right)}{2 m}
$$

( $\gamma=0$ for $P=P_{c}$ leads to the autonomous equation (4.2)), so that $\gamma<0$ if $P>P_{C}$ and the nonlinear term forms an exponentially small perturbation of the linear equation (3.6). This implies the existence of global small solutions regardless of the sign of the nonlinear term $g$, (see [10, 18], cf. a general semigroup approach in [7]). 
The asymptotic behavior is then expected to be "almost" the same as for the linear equation (3.6) (see comments below on special critical cases). For $g(v)=-|v|^{p}$ with $p>p_{c}=1+2 m / N$, the generic stable behavior $v(y, \tau)=$ $C_{0} \psi_{0}(y)+o(1)$ as $\tau \rightarrow \infty$ was established in [10]. A dynamical system approach there admits extensions to more general equations.

4.2. On stable similarity solutions in the subcritical range. Let $P \in\left(1, P_{c}\right)$. In view of (1.4), we perform the rescaling corresponding to the invariant group of transformations

$$
u=(1+t)^{\mu} v, \quad y=\frac{x}{(1+t)^{1 / 2 m}}, \quad \tau=\ln (1+t): \mathbb{R}_{+} \longrightarrow \mathbb{R}_{+},
$$

with the negative exponent $\mu=-(1+Q / 2 m) /(P-1)$ for $Q>-2 m$. This gives the autonomous equation for the rescaled solution

$$
v_{\tau}=\mathbf{B}_{*} v-g(y, v) \text { for } \tau>0
$$

where

$$
\mathbf{B}_{*}=\mathbf{B}+c_{*} I, \quad c_{*}=\frac{N\left(P_{C}-P\right)}{2 m(P-1)}
$$

with spectrum $\sigma\left(\mathbf{B}_{*}\right)=\left\{c_{*}-|\beta| / 2 m\right\}$. Hence, $c_{*}>0$ for $P \in\left(1, P_{c}\right)$, operator $\mathbf{B}_{*}$ in (4.15) has finite positive Morse index and $v \equiv 0$ is unstable stationary solution (unlike the case $P \geq P_{c}$ ). This suggests looking for a nontrivial similarity profiles $V=V(y)$ satisfying the stationary elliptic equation

$$
\mathbf{B}_{*} V-g(y, V)=0 \quad \text { in } \mathbb{R}^{N}
$$

with exponential decay as $y \rightarrow \infty$. Such very singular similarity solutions (VSS) describing the generic asymptotic behavior as $t \rightarrow \infty$ are known from the 1980s for the second-order $(m=1)$ semilinear equations with $g(v)=v^{p}$; see first results on existence, uniqueness, and stability of a similarity profile $V>0$ in $[3,16,26]$. For higher-order equations with $m>1$, these interesting problems remain open. For a particular equation (1.6) with $1<p<1+2 m / N$, some analytical and numerical evidence of existence of a finite number of VSS's (the first one stable) is presented in [22].

4.3. On countable subset of critical exponents. It follows from (4.16) that there exists a countable subset of exponents $\left\{P_{k}\right\}$ with integer $k=|\beta|$ such that $\mathbf{B}_{*}$ has a nontrivial centre subspace,

$$
c_{*}-\frac{k}{2 m}=0 \Longrightarrow P_{k}=1+\frac{2 m+Q}{k+N}, \quad k=0,1,2, \ldots,
$$

and hence, $P_{c}$ is the first one $P_{0}$ with $k=0$. In the radial setting, assuming that $g=g(|x|, u)$, for arbitrary even $k=2,4, \ldots$, operator $\mathbf{B}_{*}$ has eigenvalue 0 with a one-dimensional centre subspace $E^{c}=\operatorname{Span}\left\{\psi_{k}(|y|)\right\}$ and a finite number 
of isolated positive eigenvalues. The rest of the construction is quite similar to that for $k=0$ in Theorem 4.1. Assuming that a centre manifold analysis applies in this case (though it is more delicate) and looking for a solution of (4.15) in the form $v(\tau)=a_{k}(\tau) \psi_{k}(y)+\cdots$, we obtain the asymptotic ODE $a_{k}^{\prime}=-c_{k}\left|a_{k}\right|^{P-1} a_{k}+\cdots$ with the coefficient $c_{k}=\left\langle g\left(\cdot, \psi_{k}\right), \psi_{k}^{*}\right\rangle$ assumed to be positive. For $c_{k}<0$, the centre manifold behavior is unstable. This gives the orbit $a_{k}(\tau)= \pm C_{k} \tau^{-1 /(P-1)}+\cdots$ as $\tau \rightarrow \infty$, where $C_{k}=\left[c_{k}(2 m+Q) /(k+\right.$ $N)]^{-(k+N) /(2 m+Q)}$. Finally, returning back to the original variables via (4.14), we derive the following asymptotic patterns in the critical cases $P=P_{k}$ for even $k=2,4, \ldots$ (cf. (1.12)):

$$
u(x, t)= \pm C_{k} t^{-(k+N) / 2 m}(\ln t)^{-(k+N) /(2 m+Q)} \psi_{k}\left(\frac{|x|}{t^{1 / 2 m}}\right)+\cdots \quad \text { for } t \gg 1 .
$$

The first term of such asymptotic behavior does not reveal any trace of initial data. We again obtain $\ln t$-perturbed asymptotic patterns at the countable subset of critical exponents $P=P_{k}$. In the case of nonlinearity $g(x, v)=-|v|^{p}$, spectra of asymptotically exponentially decaying patterns for (1.7) including the critical cases were studied in [10]. A countable subset of logarithmically perturbed patterns can be constructed for nonlinear reaction-absorption equations (1.16) and (1.22) (see [14]).

ACKNOWLEDGMENT. This research was supported by RTN network HPRNCT-2002-00274 and by the INTAS project CEC-INTAS-RFBR96-1060.

\section{REFERENCES}

[1] F. Bernis, J. Hulshof, and J. L. Vázquez, A very singular solution for the dual porous medium equation and the asymptotic behaviour of general solutions, J. Reine Angew. Math. 435 (1993), 1-31.

[2] M. S. Birman and M. Z. Solomjak, Spectral Theory of Selfadjoint Operators in Hilbert Space, Mathematics and Its Applications (Soviet Series), D. Reidel Publishing, Dordrecht, 1987.

[3] H. Brezis, L. A. Peletier, and D. Terman, A very singular solution of the heat equation with absorption, Arch. Rational Mech. Anal. 95 (1986), no. 3, 185-209.

[4] J. Bricmont and A. Kupiainen, Stable non-Gaussian diffusive profiles, Nonlinear Anal. 26 (1996), no. 3, 583-593.

[5] J. Bricmont, A. Kupiainen, and G. Lin, Renormalization-group and asymptotics of solutions of nonlinear parabolic equations, Comm. Pure Appl. Math. 47 (1994), no. 6, 893-922.

[6] M. Chaves and V. A. Galaktionov, Regional blow-up for a higher-order semilinear parabolic equation, European J. Appl. Math. 12 (2001), no. 5, 601-623.

[7] S. Cui, Local and global existence of solutions to semilinear parabolic initial value problems, Nonlinear Anal., Ser. A: Theory Methods 43 (2001), no. 3, 293323.

[8] Ju. L. Dalec'kiǔ and M. G. Kreĭn, Stability of Solutions of Differential Equations in Banach Space, Translations of Mathematical Monographs, vol. 43, American Mathematical Society, Rhode Island, 1974. 
[9] Yu. V. Egorov, V. A. Galaktionov, V. A. Kondratiev, and S. I. Pohozaev, On the necessary conditions of global existence to a quasilinear inequality in the half-space, C. R. Acad. Sci. Paris Sér. I Math. 330 (2000), no. 2, 93-98.

[10] - On the asymptotics of global solutions of higher-order semilinear parabolic equations in the supercritical range, C. R. Math. Acad. Sci. Paris 335 (2002), no. $10,805-810$.

[11] S. D. Ė̀del'man, Parabolic Systems, Translated from the Russian by Scripta Technica, London, North-Holland Publishing, Amsterdam, 1969.

[12] A. Friedman, Partial Differential Equations, Robert E. Krieger Publishing, Florida, 1983.

[13] V. A. Galaktionov, On a spectrum of blow-up patterns for a higher-order semilinear parabolic equation, R. Soc. Lond. Proc. Ser. A Math. Phys. Eng. Sci. 457 (2001), no. 2011, 1623-1643.

[14] V. A. Galaktionov and P. Harwin, Spectra of critical exponents in nonlinear heat equations with absorption, submitted to Int. J. Free Boundaries.

[15] V. A. Galaktionov, S. P. Kurdyumov, and A. A. Samarskii, Asymptotic "eigenfunctions" of the Cauchy problem for a nonlinear parabolic equation, Math. USSR Sbornik 54 (1985), 421-455.

[16] On asymptotic stability of self-similar solutions of the heat equation with a nonlinear sink, Soviet Math. Dokl. 31 (1985), 271-276.

[17] V. A. Galaktionov and H. A. Levine, A general approach to critical Fujita exponents in nonlinear parabolic problems, Nonlinear Anal. 34 (1998), no. 7, 10051027.

[18] V. A. Galaktionov and S. I. Pohozaev, Existence and blow-up for higher-order semilinear parabolic equations: majorizing order-preserving operators, Indiana Univ. Math. J. 51 (2002), no. 6, 1321-1338.

[19] V. A. Galaktionov and S. A. Posashkov, An approximate self-similar solution of a nonlinear equation of heat conduction with absorption (Moscow, 1984), Mathematical Modeling, Nauka, Moscow, 1989, pp. 103-122 (Russian).

[20] V. A. Galaktionov, S. A. Posashkov, and J. L. Vázquez, Asymptotic convergence to dipole solutions in nonlinear parabolic equations, Proc. Roy. Soc. Edinburgh Sect. A 125 (1995), no. 5, 877-900.

[21] V. A. Galaktionov and J. L. Vázquez, Asymptotic behaviour of nonlinear parabolic equations with critical exponents. A dynamical systems approach, J. Funct. Anal. 100 (1991), no. 2, 435-462.

[22] V. A. Galaktionov and J. F. Williams, On very singular similarity solutions of a higher-order semilinear parabolic equation, to appear in Anal. and Appl.

[23] A. Gmira and L. Véron, Large time behaviour of the solutions of a semilinear parabolic equation in $\mathbf{R}^{N}$, J. Differential Equations 53 (1984), no. 2, 258276.

[24] I. Gohberg, S. Goldberg, and M. A. Kaashoek, Classes of Linear Operators. Vol. I, Operator Theory: Advances and Applications, vol. 49, Birkhäuser Verlag, Basel, 1990.

[25] S. Kamin and M. Ughi, On the behaviour as $t \rightarrow \infty$ of the solutions of the Cauchy problem for certain nonlinear parabolic equations, J. Math. Anal. Appl. 128 (1987), no. 2, 456-469.

[26] S. Kamin and L. Véron, Existence and uniqueness of the very singular solution of the porous media equation with absorption, J. Analyse Math. 51 (1988), 245-258.

[27] A. N. Kolmogorov and S. V. Fomin, Elements of the Theory of Functions and Functional Analysis, Izdat. Nauka, Moscow, 1976. 
[28] A. Lunardi, Analytic Semigroups and Optimal Regularity in Parabolic Problems, Progress in Nonlinear Differential Equations and Their Applications, vol. 16, Birkhäuser Verlag, Basel, 1995.

[29] A. Pazy, Semigroups of Linear Operators and Applications to Partial Differential Equations, Applied Mathematical Sciences, vol. 44, Springer-Verlag, New York, 1983.

[30] L. A. Peletier and W. C. Troy, Spatial Patterns. Higher Order Models in Physics and Mechanics, Progress in Nonlinear Differential Equations and Their Applications, vol. 45, Birkhäuser Boston, Massachusetts, 2001.

[31] A. A. Samarskii, V. A. Galaktionov, S. P. Kurdyumov, and A. P. Mikhailov, Blow-up in Quasilinear Parabolic Equations, de Gruyter Expositions in Mathematics, vol. 19, Walter de Gruyter, New york, 1995.

[32] G. R. Sell and Y. C. You, Inertial manifolds: the nonselfadjoint case, J. Differential Equations 96 (1992), no. 2, 203-255.

[33] M. E. Taylor, Partial Differential Equations. III. Nonlinear Equations, Applied Mathematical Sciences, vol. 117, Springer-Verlag, New York, 1997.

Victor A. Galaktionov: Keldysh Institute of Applied Mathematics, Miusskaya Square 4, 125047 Moscow, Russia; Department of Mathematical Sciences, University of Bath, Bath BA2 7AY, UK

E-mail address: vag@maths . bath.ac.uk 


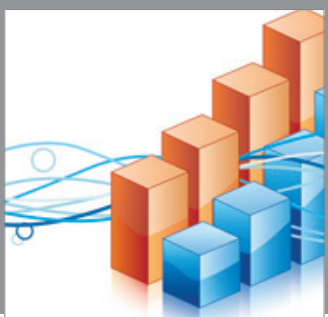

Advances in

Operations Research

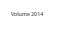

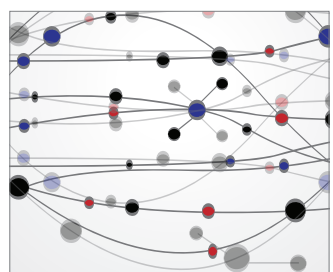

\section{The Scientific} World Journal
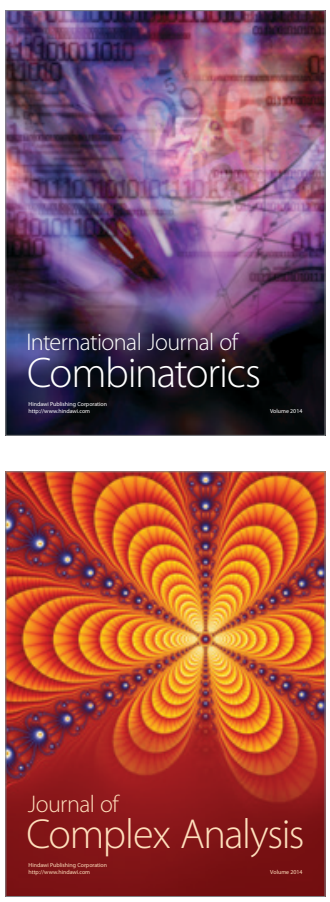

International Journal of

Mathematics and

Mathematical

Sciences
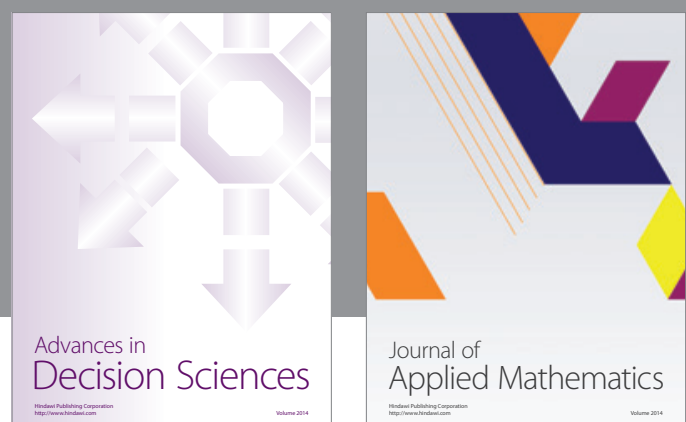

Journal of

Applied Mathematics
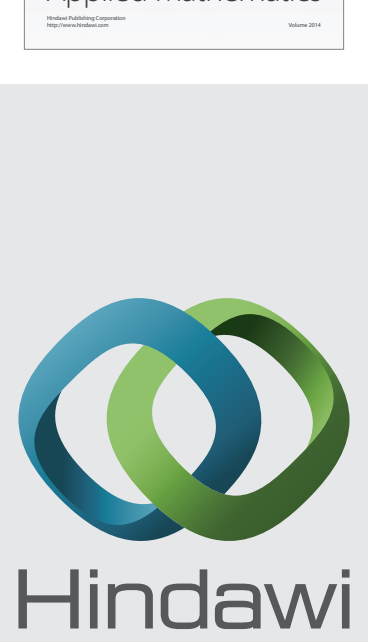

Submit your manuscripts at http://www.hindawi.com
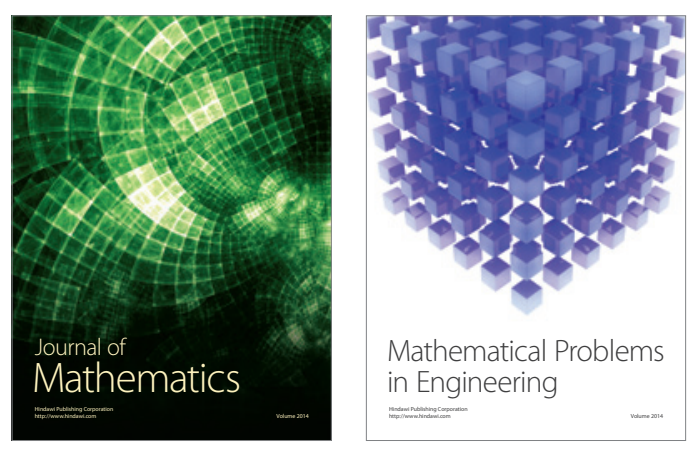

Mathematical Problems in Engineering
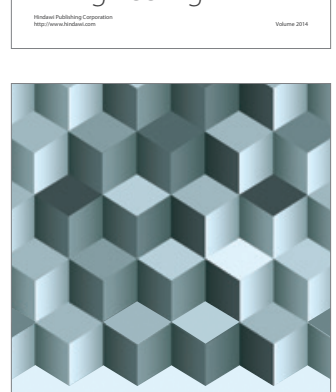

Journal of

Function Spaces
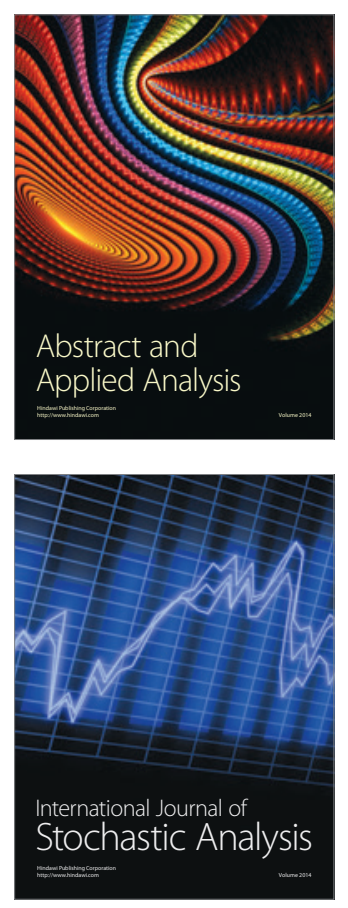

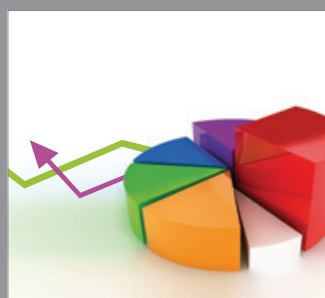

ournal of

Probability and Statistics

Promensencen
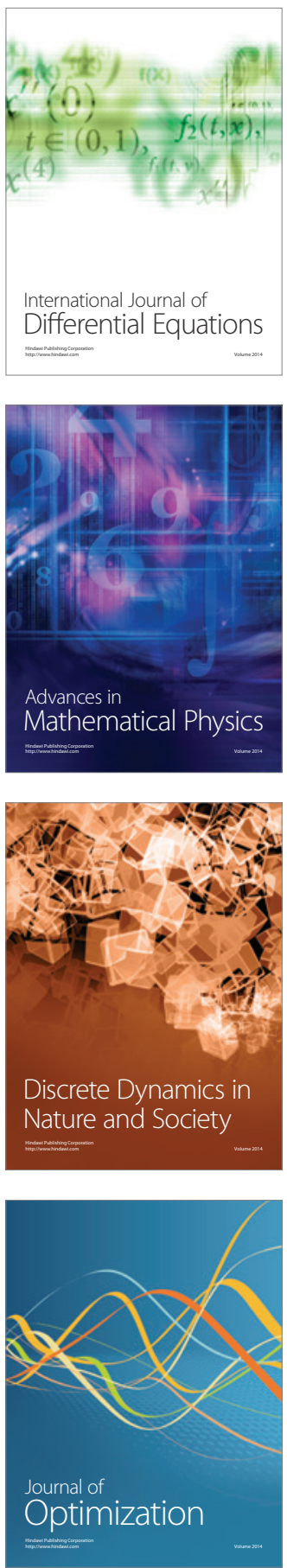